\title{
Mouse killing and carrying by Maudsley and Long-Evans strain rats
}

\author{
DANIEL J. LONOWSKI, ROBERT A. LEVITT, and SCOTT D. LARSON \\ Southern Ilinois University, Carbondale, Illinois 62901
}

The incidence of mouse killing and mouse carrying in three strains of rats was investigated. Both sexes of the Maudsley reactive, Maudsley nonreactive, and Long-Evans rats served as subjects. The highest incidence of mouse killing was observed among male reactives $(44 \%)$, an intermediate incidence in Long-Evans males (24\%), and a low incidence in the Maudsley nonreactive males $(8 \%)$. Female reactives, female Long-Evans, and female nonreactives showed similar low incidences of killing $(14 \%, 13 \%$, and $11 \%$, respectively). Male and female nonreactives and female reactives showed the highest percentages of mouse carrying $(78 \%, 87 \%$, and $81 \%$, respectively) with male reactives at an intermediate percentage $(48 \%)$ and Long-Evans males and females at the lowest percentage (28\% and $26 \%)$. The possible relationship between selective breeding for open-field reactivity and the incidence of mouse killing is discussed.

Mouse killing by rats is regarded as an example of predatory aggression. The mouse-killing response is a highly stereotyed and consistent behavior. Upon presentation of a mouse, the rat will suddenly seize the mouse, arch its back, hold the mouse with all four paws, and with successive bites directed at the cervical spine, complete the kill.

Despite the high degree of predictability for the occurrence of mouse killing, approximately $80 \%$ of laboratory rats do not kill mice (Karli, 1956; Bandler \& Moyer, 1970; Lonowski, Levitt, \& Larson, 1973).Among these nonkiller rats, however, are found rats which will carry mice. Mouse carrying resembles pup carrying and retrieval in that mice are picked up by the skin and carried to a distant area of the cage. Mouse carrying can be observed in male and female rats, and its frequency may range from 1 to 60 carries in a 15-min test session (Lonowski, Levitt, \& Larson, 1973).

Why some rats kill mice while others do not and why some rats carry mice is far from clear. There have been, however, attempts to identify various factors which may contribute to the occurrence of mouse killing. It has been shown that mouse killing in female rats is not related to gestation, parturition, or lactation (Baenninger, 1969); competition for food as well as food deprivation increases the frequency of mouse killing in nonkiller rats (Heimstra, 1965; Paul, Miley, \& Baenninger, 1971); and electric shock contingent on mouse killing suppresses the behavior (Myer \& Baenninger, 1966). The possibility of strain differences in killing behavior has also been studied. Bandler and Moyer (1970) showed a greater frequency of chick kills in Long-Evans hooded rats compared to Sprague-Dawley albino rats, although these strains did not differ in the frequency of mouse killing.

The issue of strain differences as a critical variable in the phenomenon of mouse killing has been relatively ignored. We therefore selected two strains of laboratory rats which have been shown to differ markedly on several response dimensions: The Maudsley reactive and nonreactive strains. The Maudsley strains were selectively bred for high and low open-field reactivity (Broadhurst, 1960). A comparison of the two strains shows that Maudsley reactives ambulate less and defecate more in an open-field test apparatus (Harrington, 1971), show a lower level of activity in a rotating cage (Harrington, 1971b), show slower emergence into an unfamiliar environment, and have faster escape speeds when exposed to painful shock (Gray, 1971).

Since the Maudsley strains have been shown to diverge on several response dimensions, it was our aim to ascertain if a similar differentiation among the strains might be found for mouse killing and mouse carrying. In order to compare the behavior of these strains to one commonly used in mouse killing experiments, the Long-Evans strain was also studied.

\section{METHOD}

\section{Subjects}

A total of 189 adult rats were used as experimental subjects: 22 male and 37 female Maudsley reactives, 15 male and 15 female Maudsley nonreactives, and 50 male and 50 female Long-Evans rats. Each animal was housed individually with food and water available ad lib. In addition, each rat was handled for 1 min each day for 5 days prior to behavioral tests with mice. Approximately 100 male and female adult albino mice were used as test stimuli. All mice were housed in groups of six and were kept isolated from each of the rat strains selected for study.

\footnotetext{
Apparatus and Testing Procedure

Behavioral tests were conducted in standard-sized metal cages which also served as home cages for the rats. At the beginning of each test session, naive albino mice were randomly selected and placed in each rat's home cage. The behavior of each rat was observed and recorded for $15 \mathrm{~min}$. At the conclusion of the 15-min test, mice were removed from the test cages. A behavioral classification of mouse killer and mouse carrier was determined by
} 
the occurrence of either a kill or a minimum of one carry response during each test. Tests of this kind were conducted at the same time of day on each of 3 successive test days.

\section{RESULTS}

\section{Mouse Killing}

Figure 1 shows the average percentage of mouse killing recorded for each group of animals on the 3 successive test days. As seen in the figure, varying degrees of mouse killing were observed. The incidence of mouse killing was highest among male reactive rats $(44 \%)$ which differed from the percentage of mouse killing observed in both the Long-Evans males $(28 \%$; $\mathrm{Z}=3.37 . \mathrm{p}<.001)$ and the Maudsley nonreactive males $(8 \% ; Z=3.79 . p<.001)$. The percentage of kills also differed between the Long-Evans males and the male nonreactives $(Z=3.78, p<.001)$. The clear separation of males among the three strains, however, was not observed among the female rats, which did not differ from each other in percentage of kills. A comparison of the incidence of mouse killing within each strain showed significant sex differences among reactive and Long-Evans rats $(\mathrm{Z}=4.33, \mathrm{p}<.001$; $\mathrm{Z}=2.45, \mathrm{p}<.025)$. A comparable significant sex difference in percentage of kills was not found for the Maudsley nonreactive strain.

\section{Mouse Carrying}

Figure 2 shows the average percentage of mouse carrying for each test strain on the 3 successive days. Among the males, mouse carrying was noted to be the highest in the nonreactives $(78 \%)$, intermediate in the reactives $(48 \%)$. and lowest in the Long-Evans strain $(28 \%)) Z=2.71, \quad p<.001 ; \quad Z=8.32, \quad p<.001$; $\mathrm{Z}=3.21 . \mathrm{p}<.001)$. Among the females, reactives and nonreactives carried mice equally often $(81 \%$ and

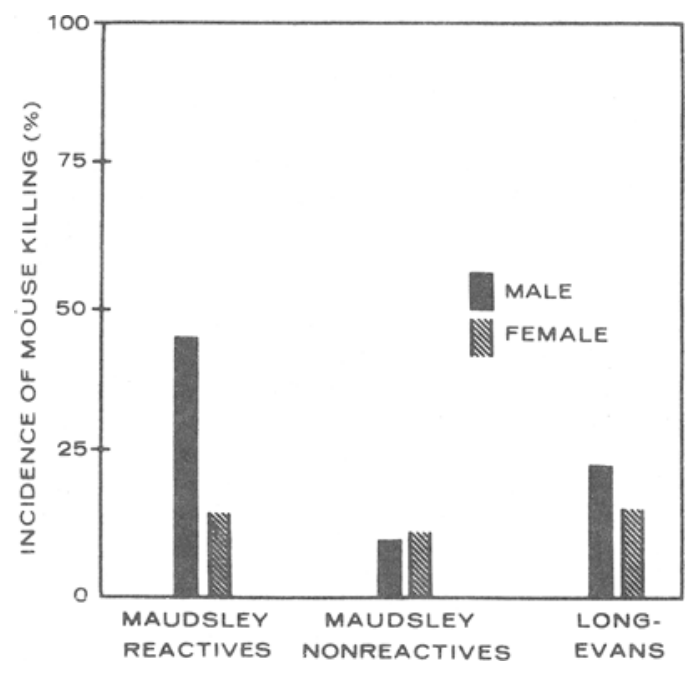

Figure 1. Incidence of mouse killing in three strains of rats.

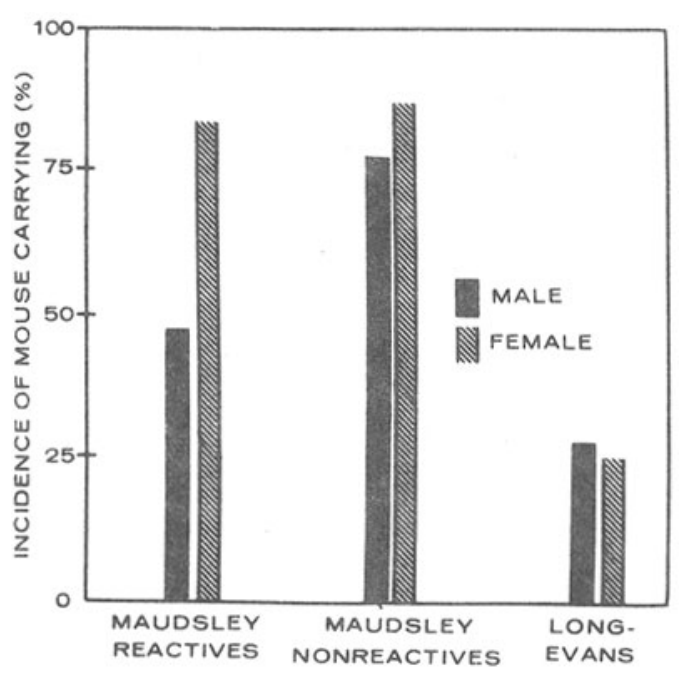

Figure 2. Incidence of mouse carrying in three strains of rats.

$86 \%$ ) and showed a higher percentage of mouse carrying than the Long-Evans rats $(26 \% ; Z=4.01$, $p<.001 ; \quad Z=3.92, \quad p<.001)$. Comparing the incidence of mouse carrying within each strain showed a significant sex difference for the reactive strain $(Z=4.02, p<.001)$. No such sex differences were found among the nonreactive or Long-Evans strains.

\section{Overall Incidence of Behavior}

It is interesting to note that $46 \%$ the Long-Evans rats engaged in either mouse killing or carrying. This finding may be contrasted to the $93 \%$ of reactives and $91 \%$ of nonreactives engaging in either mouse killing or mouse carrying $(Z=15.4, p<.001 ; Z=5.18$. $\mathrm{p}<.001)$.

\section{DISCUSSION}

The incidence of mouse killing and carrying by rats was found to differ according to the strain and sex of the rats used in this experiment. Male Maudsley reactives showed the highest incidence of killing. followed by male Long-Evans rats. Females of the reactive and Long-Evans strains and both males and females of the nonreactive strain showed similar low incidences of mouse killing (Figure 1). The incidence of mouse carrying was highest in both sexes of the nonreactive strain and in the female reactives. Maudsley reactive males showed an intermediate incidence of carrying, followed by both sexes of the Long-Evans strain, which showed the lowest incidence of carrying (Figure 2).

It is interesting to note that carrying appears to be a more predominant response than killing. The incidence of carrying ranged from about $80 \%$ to $90 \%$ in both sexes of Maudsley nonreactives and female reactives to $45 \%$ in male reactives to about $25 \%$ in both sexes of Long-Evans rats. Killing incidences, in contrast, ranged only from about $45 \%$ in male reactives to $25 \%$ in male Long-Evans to about $10 \%$ to $15 \%$ in both sexes of nonreactives and female reactive and Long-Evans rats. The incidence of killing reported in previous studies has been about 20\% (Karli, 1956; Bandler \& Moyer, 1970; Lonowski, Levitt, \& Larson. 1973). Only the incidence of killing in male reactives differed strikingly from these previous data. 
According to these data, the incidences of the killing and carrying behavior exhibited by rats toward mice differ somewhat across strain and sex. It is worthwhile to point out that the strain differences were accentuated in the males. The Maudsley strains have been investigated in a variety of situations (Broadhurst, 1960; Gray, 1971; Harrington, 1971a, b). Because of the wide range of behavioral differences which have been reported between the reactive and nonreactive strains, it has been suggested that selective breeding for open-field reactivity over several generations has produced some general attribute of behavior in these rats. It has been further proposed that these attributes may related to the level of responsiveness to novel or stressful stimuli (Gray, 1971). Accordingly, Maudsley reactives have been shown to freeze, crouch, and defecate more than nonreactives when a novel stimulus is presented. Our data is consistent with this notion of genetic behavioral differences among these rat strains along a continuum of reactivity or aggressivity.

\section{REFERENCES}

BAENNINGER, R. Independence of parturition and mouse-killing by female rats. Psychonomic Science, 1969, 15, 144.

BANDler, R. J., \& MOYer, K. E. Animals spontaneously killed by rats. Communications in Behavioral Biology, 1970, 5, 177-182.

Broadhurst, P. L. Applications of biometrical genetics to the inheritance of behavior. In H. J. Eysenck (Ed.), Experiments in personality. London: Routledge \& Kegan Paul, 1960.
GRAY, J. The psychology of fear and stress. New York: McGraw-Hill, 1971.

HARRINGTON, G. M. Strain differences in rotating wheel activity of the rat. Psychonomic Science, 1971, 23, 363-364. b

Heimstra, N. W. A further investigation of the development of mouse killing in rats. Psychonomic Science, 1965, 2, 179-180.

KARLI, P. The Norway rat's killing response to the white mouse. Behaviour, 1956, 10, 81-103.

Lonowski, D. J., LevitT, R. A., \& LaRson, S. D. Mouse killing or carrying by male and female Long-Evans hooded rats. Bulletin of the Psychonomic Society, 1973, 1, 349-351.

Myer, J. S., \& BaEnNinger, R. Some effects of punishment and stress on mouse killing by rats. Journal of Comparative and Physiological Psychology, 1966, 62, 292-297.

Paul, L., Miley, W. M., \& Baenninger, R. Mouse killing by rats: Roles of hunger and thirst in its initiation and maintenance. Journal of Comparative and Physiological Psychology, 1971, 76, 242-249.

(Received for publication September 15, 1975.) 